Ann. Zootech., 1987, 36 (3), 237-248

\title{
Induction hormonale de la lactation chez la vache: résultats de trois années d'expérimentation en ferme
}

\author{
J. PONT et C. DELOUIS (1) \\ INRA, Laboratoire de Physiologie de la Lactation \\ Centre de Recherches de Jouy-en-Josas \\ F 78350 Jouy-en-Josas
}

\begin{abstract}
Résumé
Trois cent soixante-trois vaches laitières non gestantes ont été induites en lactation à l'aide d'un traitement hormonal de courte durée dans différentes fermes de plusieurs régions de France.

Soixante-seize pour cent des vaches traitées ont une production laitière minimum de $10 \mathrm{~kg} /$ jour, mais seulement $43 \%$ des animaux ont poursuivi une lactation d'une durée supérieure à 200 jours, produisant $69 \%$ de la quantité totale de lait obtenue au cours de la lactation normale précédente. Les productions laitières totales après induction sont significativement corrélées avec celles enregistrées pour 46 animaux au cours de la lactation normale qui précède $(r=0,38$, $\mathrm{P}<0,02)$. Les taux en protéines des laits collectés au cours de la lactation induite sont significativement plus élevés que ceux des laits obtenus au cours de la lactation normale précédente de 43 vaches $(3,34$ et $3,11 \%$, respectivement, $\mathrm{P}<0,01)$. Les taux butyreux des mêmes laits ne sont pas significativement différents.

Les traitements hormonaux d'induction de la lactation réalisés entre janvier et juillet assurent un taux de réussite et une production laitière totale significativement plus élevés que lorsqu'ils sont pratiqués entre août et décembre ( 90 et $71 \% ; 4242$ et $3405 \mathrm{~kg} / 305$ jours, respectivement).

Bien que les vaches aient été induites en lactation à la suite de difficultés de reproduction, $38 \%$ des animaux sont devenus gravides pendant la lactation induite, puis ont vélé.

L'induction hormonale de la lactation peut donc être utilisée pour placer dans une situation économique plus rentable une vache à haut potentiel laitier non gravide en fin de lactation. Toutefois, à l'heure actuelle, la législation très stricte concernant l'utilisation des cestrogènes s'oppose au développement de la technique dans les conditions de la pratique.
\end{abstract}

Mots clés : Lactation induite, reproduction, vache laitière, production laitière, essais en ferme.

\section{Introduction}

L'augmentation des performances laitières du cheptel bovin s'accompagne presque toujours d'une baisse globale du potentiel de reproduction. A partir de l'analyse de près de 60000 données, OlDs et al. (1979) montrent qu'au-dessus d'une production laitière de $1000 \mathrm{~kg}$ à 120 jours de lactation, chaque fraction supplémentaire de $700 \mathrm{~kg}$ de lait correspond à l'augmentation de 0,1 du nombre d'inséminations nécessaires pour obtenir une gestation; ainsi le taux de conception en première lactation est de 70 et $45 \%$ pour des productions laitières totales à 120 jours de 1000 et $6000 \mathrm{~kg}$ respective-

(1) Demande de tiré à part : INRA, Station centrale de Physiologie animale, 78350 Jouy-en-Josas, France. 
ment. En France, ce phénomène se traduirait par des pertes économiques importantes dues à l'allongement de l'intervalle entre lactations et la réforme prématurée de vaches laitières à haut potentiel.

Chez les Ruminants, l'induction de la lactation en dehors d'un cycle de reproduction par des traitements hormonaux a été expérimentée depuis très longtemps (Mertes, 1961). Pendant de nombreuses années, les faibles productions laitières obtenues à l'aide de traitements de longue durée (60 à 80 jours) n'ont pas conduit à des applications pratiques. Plus récemment, des traitements hormonaux de courte durée ( 7 jours) ont donné des résultats très supérieurs (Smith \& Schanbacher, 1973 ; Delouis et al., 1978 ; FulKerson, 1978 ; FiEld et al., 1979) ouvrant ainsi la voie pour des applications au niveau des élevages.

L'utilisation de l'induction hormonale de la lactation peut devenir une solution complémentaire pour la gestion des vaches à haut potentiel laitier et subfertiles. Il paraissait donc intéressant de mesurer l'efficacité de la méthode d'induction de la lactation dans les conditions de la pratique au cours de plusieurs campagnes successives.

\section{Matériel et méthodes}

Trois cent soixante-trois vaches de bon niveau de production laitière (tabl. 1) non gravides en fin de lactation ont reçu un traitement d'induction. Ces animaux faisaient partie de troupeaux présents dans des exploitations de plusieurs régions de France: Maine, Mayenne, Côtes-du-Nord, Finistère, Loire-Atlantique, Région parisienne, Centre et Sud-Ouest. Les vaches étaient en très grande majorité de race Frisonne et Frisonne croisée Holstein.

Le traitement hormonal a consisté en des injections sous cutanées d'un mélange en solution alcoolique (éthanol $95 \% \mathrm{v} / \mathrm{v}$ ) 'de 0,05 et $0,125 \mathrm{mg}$ d'œstradiol $17 \beta$ (Roussel) et de progestérone (Roussel) respectivement, par $\mathrm{kg}$ de poids vif, matin et soir, pendant 7 jours. Ce premier traitement est suivi, 12 jours après la dernière administration d'œstradiol et de progestérone, par des injections intramusculaires biquotidiennes d'hydrocortisone (Roussel), $25 \mathrm{mg}$ et, pour certains animaux, de réserpine (Sigma), $2 \mathrm{mg}$ pendant 3 à 5 jours. L'addition de réserpine au traitement favorise la montée laiteuse (BAuman et al., 1977).

La traite a commencé pour tous les animaux le $21^{\mathrm{e}}$ jour qui suit la première injection d'œstradiol et de progestérone.

\section{Résultats}

\section{A. Taux de réussite et production laitière totale}

Soixante-seize pour cent des animaux traités ont eu une production laitière dont le maximum a atteint ou dépassé $10 \mathrm{~kg}$ par jour (tabl. 1). En fait, les éleveurs n'ont conservé que $43 \%$ des vaches capables d'assurer une lactation complète et surtout d'entrer rapidement en gestation. 


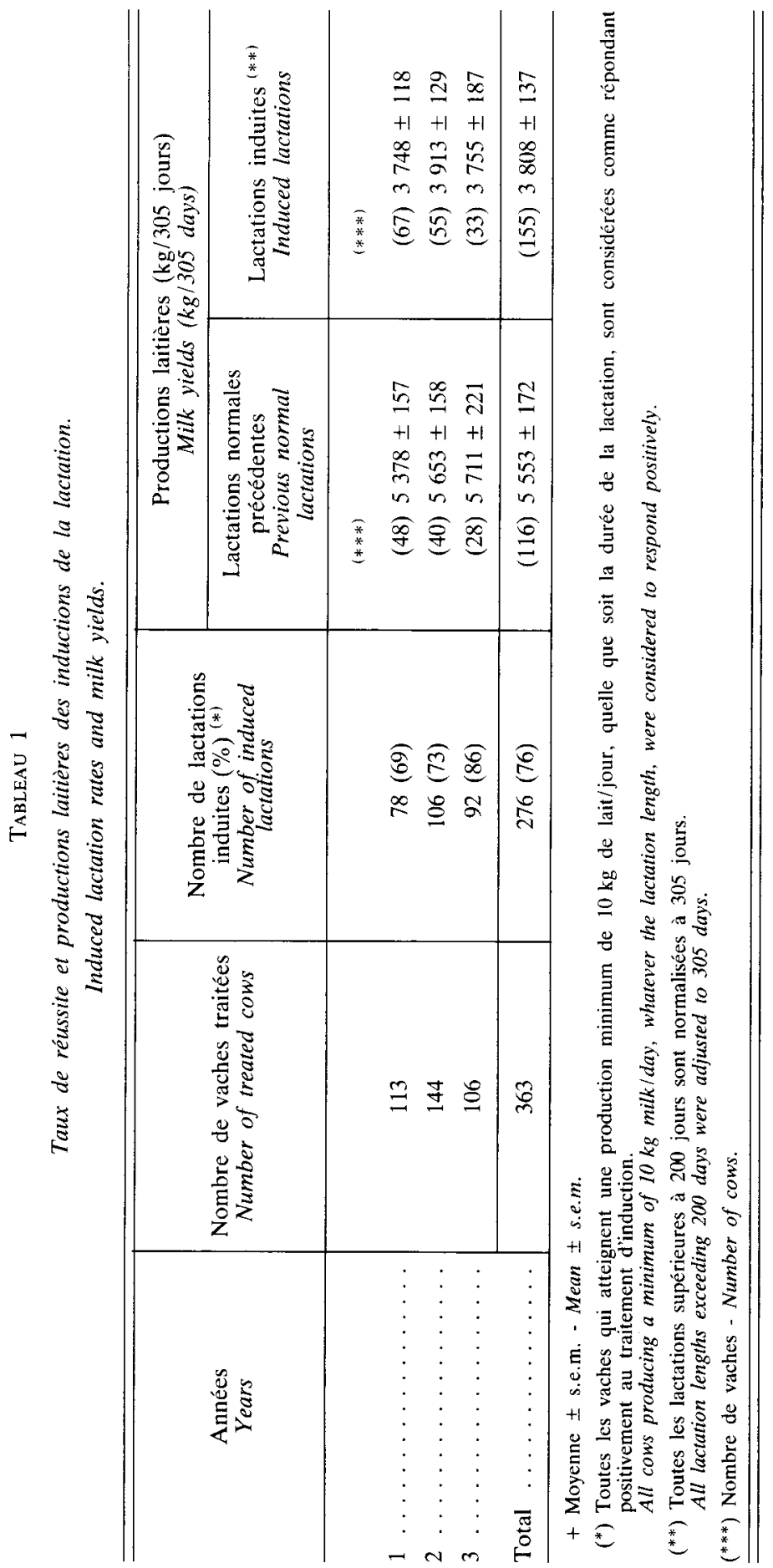


La production laitière totale pour les vaches ayant une lactation complète à été de $69 \%$ de celle obtenue au cours de la lactation précédente (tabl. 1). Il est à remarquer que cette valeur a été identique au cours des 3 campagnes $(69,69$ et $67 \%$, respectivement).

\section{B. Taux butyreux et azotés}

Les résultats concernant 43 lactations induites et normales précédentes pour les mêmes vaches ont été les suivants: taux butyreux moyens $3,90 \pm 0,06$ et $3,81 \pm 0,07 \%$ au cours des lactations induites et normales, respectivement ; taux azotés moyens $3,34 \pm 0,04$ et $3,11 \pm 0,05 \%$ au cours des lactations induites et normales, respectivement. Ainsi, les laits obtenus au cours des lactations induites ont-ils un taux azoté significativement plus élevé que celui des laits collectés au cours de la lactation normale précédente $(\mathrm{P}<0,01)$; les taux butyreux ne sont pas significativement différents.

\section{Influence de la saison}

Les traitements d'induction ont été réalisés tout au long de l'année avec deux périodes de pointe à l'automne et au printemps.

Les taux de réussite des inductions de lactation a été le plus faible lorsque les traitements ont eu lieu à l'automne lors des campagnes 1 et 3 . Cette influence négative des jours à éclairement et à températures décroissantes ne s'est pas vérifiée la deuxième année (tabl. 2). Pour l'ensemble des résultats, les traitements réalisés entre janvier et juillet ont induit $90 \%$ de vaches à produire au moins $10 \mathrm{~kg}$ lait/jour.

Les productions laitières totales ont été significativement plus élevées pour les vaches induites en lactation de janvier à juillet que pour celles induites d'août à décembre (tabl. 3). Ce résultat traduirait une influence défavorable des conditions automnales (climat, alimentation) sur la montée laiteuse après induction.

\section{Productions laitières au cours de la lactation induite et au cours de la lactation normale qui précède ou qui suit}

Quarante-six vaches ont été suivies au contrôle laitier au cours de la lactation qui a précédé et au cours de celle qui a suivi la lactation induite (tabl. 4).

La production laitière totale moyenne au cours de la lactation induite est de 70 et $61 \%$ de celle enregistrée au cours de la lactation qui précède et de celle qui suit, respectivement. Ainsi, pendant la lactation qui suit la lactation induite, la production laitière totale est-elle supérieure de $64 \%$. Cette augmentation ne peut pas s'expliquer seulement par l'augmentation normale de la production laitière au cours des 4 ou 5 premières lactations. En effet, l'augmentation de la production laitière totale au cours de la lactation qui suit la lactation induite n'est que de $13 \%$ par rapport à celle enregistrée au cours de la lactation normale qui précède (tabl. 4).

Il existe une corrélation significative entre les productions laitières obtenues au cours de 3 lactations successives chez le même animal, la lactation intermédiaire étant une lactation induite (tabl. 4). Ce résultat confirme celui rapporté précédemment sur un nombre plus faible de données (Delouis et al., 1978). 


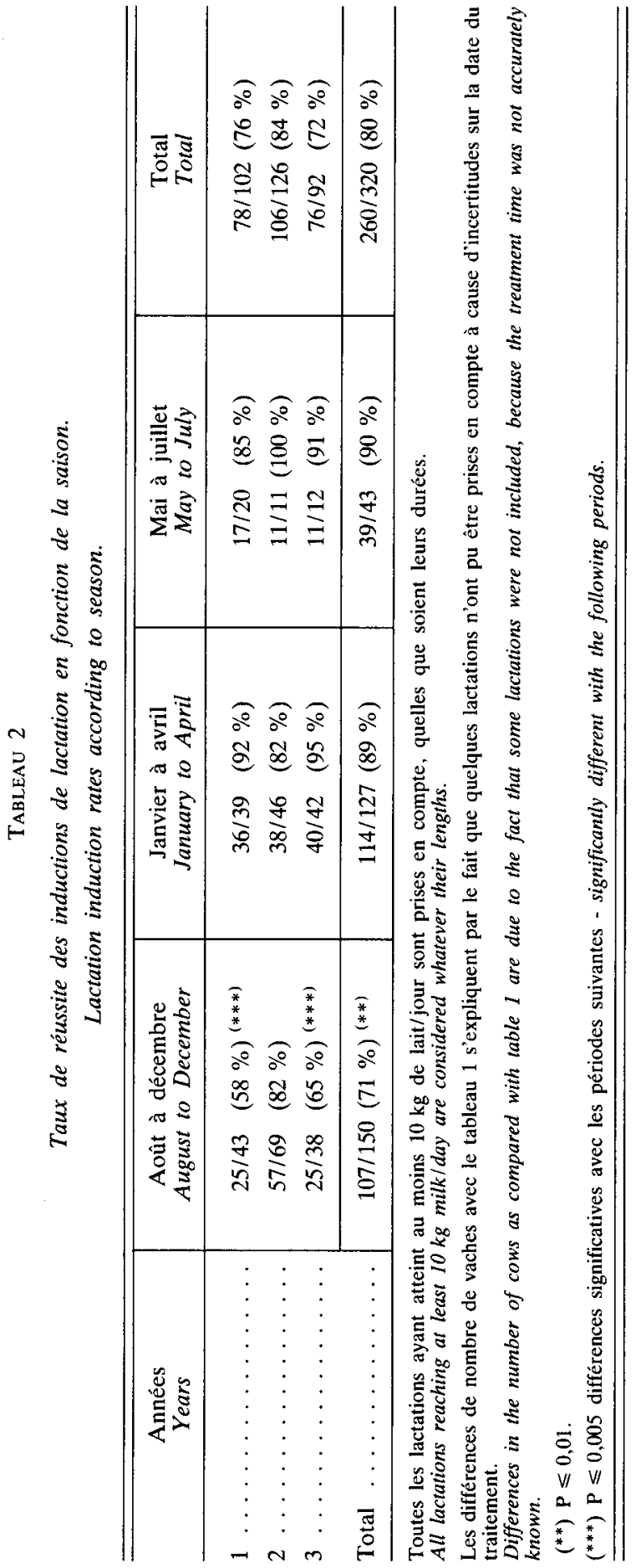




\section{TABleaU 3}

Influence de la saison sur les productions laitières totales après induction de la lactation. Influence of the season on milk yields during induced lactations.

\begin{tabular}{|c|c|c|c|c|}
\hline \multirow{2}{*}{$\begin{array}{l}\text { Années } \\
\text { Years }\end{array}$} & \multicolumn{2}{|c|}{$\begin{array}{l}\text { Août à décembre } \\
\text { August to December }\end{array}$} & \multicolumn{2}{|c|}{$\begin{array}{l}\text { Janvier à juillet } \\
\text { January to July }\end{array}$} \\
\hline & $\begin{array}{l}\text { Nombre } \\
\text { Number }\end{array}$ & $\begin{array}{l}\text { Moyenne } \\
\text { Mean }\end{array}$ & $\begin{array}{l}\text { Nombre } \\
\text { Number }\end{array}$ & $\begin{array}{l}\text { Moyenne } \\
\text { Mean }\end{array}$ \\
\hline 1 & 25 & $3223^{+b} \pm 146^{(*)}$ & 32 & $4224^{\mathrm{b}} \pm 202$ \\
\hline 2 & 31 & $3691^{\mathrm{a}} \pm 184$ & 19 & $4336^{a} \pm 241$ \\
\hline 3 & 10 & $2976^{\mathrm{b}} \pm 200$ & 15 & $4160^{b} \pm 226$ \\
\hline
\end{tabular}

a Différence significative à $\mathrm{P}<0,05$ - Significantly different $P<0.05$.

b Différence significative à $\mathrm{P}<0,01$ - Significantly different $P<0.01$.

+ Production laitière $(\mathrm{kg} / 305$ jours) - Milk yield ( $\mathrm{kg} / 305$ days).

$\left({ }^{*}\right)$ s.e.m.

\section{Tableau 4}

Comparaisons entre productions laitières au cours de la lactation induite et au cours de la lactation normale qui la précède et qui la suit.

Comparisons between milk yields during induced lactation and during previous and following normal lactation.

\begin{tabular}{|c|c|c|c|c|c|}
\hline & \multirow[t]{2}{*}{$\begin{array}{c}\text { Moyenne } \\
\text { Mean }\end{array}$} & \multicolumn{4}{|c|}{$\begin{array}{c}\text { Correlations }(n=46) \\
\text { Correlations }\end{array}$} \\
\hline & & & PL & PI & PS \\
\hline PL & $5484^{+} \pm 116^{++}$ & PL $\ldots$ & 1 & & \\
\hline PI & $3857 \pm 135$ & PI . & $0,38^{(*)}$ & 1 & \\
\hline PS $\ldots \ldots$ & $6338 \pm 197$ & PS $\ldots$. & $0,50^{(* *)}$ & $0,39^{(*)}$ & 1 \\
\hline
\end{tabular}

PL Production laitière pendant la lactation précédente - Milk yield during previous lactation.

PI Production laitière pendant la lactation induite - Milk yield during induced lactation.

PS Production laitière pendant la lactation suivante - Milk yield during following lactation.

a Les moyennes sont établies à partir de 46 vaches - Means were calculated from 46 cows.

$+\mathrm{kg} / 305$ jours $-\mathrm{Kg} / 305$ days.

++ s.e.m.

$\left({ }^{*}\right)$ Significatif $\mathrm{P}<0,02$ - Significant $P<0.02$.

$\left(^{* *}\right)$ Significatif $P<0,01-$ Significant $P<0.01$.

\section{E. Fertilité après traitement d'induction de la lactation}

Cent trente-sept vaches ont eu une gestation confirmée au cours de la lactation induite ; elles représentent $38 \%$ de l'effectif total des animaux traités pendant l'ensemble de l'essai (tabl. 5a). Le potentiel de reproduction s'élève à $50 \%$ de l'effectif des vaches ayant eu une lactation induite réussie (plus de $10 \mathrm{~kg}$ de lait/jour). Globalement, le traitement d'induction de la lactation pratiqué sur des animaux non gravides en fin de lactation normale a permis de remettre dans un cycle de reproduction $38 \%$ des animaux en lactation induite. 


\section{TABLEAU 5}

Potentiel de reproduction après induction de la lactation.

Reproductive traits after induced lactation.

a) Essais en fermes. / On-the-farm trials.

\begin{tabular}{|c|c|c|c|c|}
\hline $\begin{array}{l}\text { Années } \\
\text { Years }\end{array}$ & $\begin{array}{c}\text { Nombre de vaches } \\
\text { traitées } \\
\text { Number of treated cows }\end{array}$ & $\begin{array}{c}\text { Nombre de lactations } \\
\text { indaites } \\
(>10 \mathrm{~kg} \text { lait/jour }) \\
\text { Number of induced } \\
\text { lactations } \\
(>10 \mathrm{~kg} \text { milk/day) }\end{array}$ & $\begin{array}{c}\text { Nombre de lactations } \\
\text { complètes ( }>200 \text { jours) } \\
\text { Number of complete } \\
\text { lactations }(>200 \text { days) }\end{array}$ & $\begin{array}{l}\text { Nombre de gestations } \\
\text { Number of pregnancies }\end{array}$ \\
\hline 1 & 113 & 78 & 67 & 48 \\
\hline 2 & 144 & 106 & 55 & 50 \\
\hline \multirow[t]{5}{*}{3} & 106 & 92 & 33 & 39 \\
\hline & $\overline{363}$ & 276 & 155 & 137 \\
\hline & \multicolumn{4}{|c|}{$L$} \\
\hline & \multicolumn{4}{|c|}{ L_ $50 \% \longrightarrow$} \\
\hline & \multicolumn{4}{|c|}{ 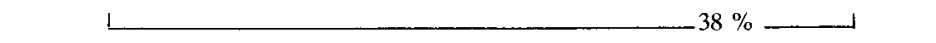 } \\
\hline
\end{tabular}

b) Essais en stations expérimentales. / In-station trials.

\begin{tabular}{|c|c|c|c|c|c|c|c|}
\hline \multirow{3}{*}{ INRA. Bressonvilliers } & \multirow{2}{*}{$\begin{array}{c}\text { Nombre } \\
\text { de } \\
\text { vaches } \\
\text { Number } \\
\text { of cows }\end{array}$} & \multirow{2}{*}{$\begin{array}{l}\text { Etat physiologique } \\
\text { avant traitcment } \\
\text { Physiological sute } \\
\text { before treatment } \\
\\
\begin{array}{l}\text { Subfertile } \\
\text { Subferile }\end{array}\end{array}$} & \multirow{2}{*}{$\begin{array}{c}\begin{array}{c}\text { Nature et doses } \\
\text { des aestrogenes } \\
\text { (mg/kg/j }\end{array} \\
\text { pendant } 7 \text { jours) } \\
\text { Type and doses } \\
\text { of oestrogens } \\
\text { (mg/ } / \mathrm{kg} / \mathrm{d} \text { for } 7 \mathrm{days}) \\
\text { (Estradiol (0.1) }\end{array}$} & \multirow{2}{*}{$\begin{array}{l}\text { Nombre } \\
\text { dc } \\
\text { vaches } \\
\text { gestantes } \\
\text { Number } \\
\text { of } \\
\text { pregnant } \\
\text { cows } \\
y\end{array}$} & \multicolumn{3}{|c|}{$\begin{array}{c}\text { Nombre moyen de jours de la lactation } \\
\text { ou nombre I.A. nécessaires pour obtenir } \\
\text { l'entrée en gestation } \\
\text { Number of days of induced lactations } \\
\text { or A.L. required for onset of pregnancy }\end{array}$} \\
\hline & & & & & 1 I.A. (5) & 2 I.A. (3) & 3 I.A. (I) \\
\hline & 15 & $\begin{array}{l}\text { Témoin non traité } \\
\text { Control, untreated }\end{array}$ & & 15 & I I.A. (13) & 2 I.A. (2) & \\
\hline \multirow[t]{2}{*}{$\begin{array}{l}\text { INRA. } \\
\text { Jouy-en-Josas . . . . . }\end{array}$} & 11 & $\begin{array}{l}\text { Primipare } \\
\text { Primipurous }\end{array}$ & (Estradiol (0.1) & 10 & $\begin{array}{c}150 \text { jours } \\
\text { days }\end{array}$ & & \\
\hline & 21 & $\begin{array}{l}\text { Mulfipare } \\
\text { Multiparous }\end{array}$ & (Estradiol (0.1) & 16 & $\begin{array}{c}120 \text { jours } \\
\text { days }\end{array}$ & & \\
\hline \multirow[t]{3}{*}{ GARCIA et al. (1980) } & 10) & $\begin{array}{l}\text { Subfertile } \\
\text { Subferille }\end{array}$ & D.E.S. ${ }^{+}(0.1)$ & y & 1 I.A. (7) & 2 I.A. (2) & \\
\hline & 9 & $\begin{array}{l}\text { Subfertile } \\
\text { Subfertile }\end{array}$ & $\begin{array}{l}\text { Benzoate diestradiol } \\
\text { d'oestradiol }^{(*)}(0.1)\end{array}$ & 8 & II.A. (6) & 2 I.A. (2) & \\
\hline & 12 & $\begin{array}{l}\text { Subfertile } \\
\text { Subfertile }\end{array}$ & CEstradiol (0.1) & 10 & I I.A. (8) & 2 I.A. (2) & \\
\hline PEEL et al. (1979) & 39 & $\begin{array}{l}\text { Normal } \\
\text { Normal }\end{array}$ & $\begin{array}{c}\text { Benzoate } \\
\text { d'cestradiol }(0.05)\end{array}$ & 35 & $N D^{(* 8)}$ & & \\
\hline $\begin{array}{l}\text { + D.E.S. = Diethylstilhes } \\
\text { (*) En equivalent uestradia } \\
\text { (*) ND = Inconnu - Unh }\end{array}$ & oestra & wivalent. & & . & & & \\
\hline
\end{tabular}


Les résultats concernant la fertilité après traitement d'induction de la lactation en ferme sont très inférieurs à ceux obtenus en stations expérimentales (tabl. 5b). Dans ce dernier cas, $85 \%$ des vaches traitées sont devenues gestantes au cours de leur lactation induite. La différence vient de ce que la réussite du traitement, en terme de niveau de production laitière, n'est pas prise en compte de la même façon. Il est évident que les éleveurs ont préférenciellement conservé en lactation induite les vaches devenues gravides, alors que les essais en station ont eu pour résultat de conserver des animaux induits en lactation.

\section{Discussion}

Les résultats de l'induction hormonale de la lactation par un traitement de courte durée, obtenus dans les conditions de la pratique, confirment ceux rapportés précédemment à partir d'essais en station (SMith \& Schanbacher, 1973 ; Collier et al., 1975 ; Delouis et al., 1978 ; Peel et al., 1978). Le pourcentage de réponses positives au traitement $(76 \%)$ peut être considéré comme satisfaisant. Toutefois, il existe de très grandes variations au niveau des élevages; dans certaines exploitations, aucune induction ne réussit; dans d'autres elles réussissent presque toutes. Ces variations sont difficiles à expliquer, compte tenu du faible nombre d'animaux par élevage.

La production laitière totale obtenue est en moyenne égale à $69 \%$ de la lactation précédente et qui sert de référence pour les vaches conservées plus de 200 jours à la traite, soit $43 \%$ de l'effectif des animaux soumis au traitement. Cette moyenne recouvre des différences très importantes (2 à $6000 \mathrm{~kg} / 305$ jours). Beaucoup d'éleveurs considèrent une production laitière inférieure à $4000 \mathrm{~kg}$ comme insuffisante.

La nature du traitement, 24 injections réparties en 2 périodes, sur 12 jours, constitue une difficulté pour le développement de la technique. Quelques essais, notamment sur chèvre (DE MonTIGNY et al., 1980) ont montré que l'injection de la dose journalière de stéroïdes en une seule fois ne modifiait pas les pourcentages de réussite. Plus récemment, Davis et al. (1983) utilisent comme mode d'administration des stéroïdes des éponges vaginales chez la vache ; les résultats sont comparables à ceux obtenus par injections sous-cutanées biquotidiennes. Toutefois, le taux de réussite est plus faible avec les éponges vaginales qu'avec les injections sous-cutanées chez la chèvre (Pont J., résultats non publiés). Une possibilité reste, évidemment, l'apport des hormones sous forme d'implant. Cette méthode pourrait être mise au point après la mise en place d'une législation autorisant l'utilisation des cestrogènes dans les conditions de la pratique.

Les essais d'amélioration du traitement d'induction ont porté sur l'ajustement des doses de stéroïdes et leur répartition au cours de ce traitement (Fulkerson, 1978 ; Peel et al., 1979). Il ne semble pas qu'une modification de la dose de stéroïdes injectés puisse apporter une amélioration des résultats. Une augmentation des doses d'œstrogènes, jusqu'à $0,2 \mathrm{mg} / \mathrm{kg}$ de poids (Delouis et al., 1978), ne modifie pas significativement les résultats et apporte davantage de perturbations du comportement des animaux. La dose de $0,05 \mathrm{mg} / \mathrm{kg}$ essayée sur bovins et sur caprins est suffisante pour déclencher les lactations du même ordre de grandeur, mais les pourcentages de réponses positives sont plus faibles (PonT J., résultats non publiés). 
La nature des œstrogènes utilisés peut jouer un rôle important (GARCIA et al., 1980). Le benzoate d'œstradiol donne le meilleur taux de réussite et les meilleures productions laitières, mais il déprime légèrement les performances de reproduction. L'étude comparée des niveaux plasmatiques hormonaux (œstrogènes totaux, prolactine) réalisée dans le cadre de ces essais montre que le taux d'œstrogènes se maintient pendant plus longtemps à un niveau élevé avec le benzoate d'œstradiol qu'avec l'œstradiol, la prolactinémie étant restée sensiblement la même pendant les deux traitements. La comparaison des effets de ce traitement avec ceux du traitement par le diéthylstilbestrol n'a pas été faite.

Pendant la montée laiteuse, il se produit une élévation plus ou moins importante de la prolactinémie (Convey, 1974 ; Delouis et al., 1978) qui, sur des animaux ayant reçu ce traitement d'induction, reste toujours très inférieure à celle observée avant le vélage. Elle est parfois très faible, ce qui correspond, en général, à des productions laitières plus faibles.

C'est pour tenter de normaliser la sécrétion de prolactine au niveau hypophysaire que les animaux reçoivent un traitement complémentaire par la réserpine (Collier et al., 1976 ; Bauman et al., 1977 ; PeEl et al., 1979 ; Johke et al., 1980). L'importance de la dose injectée, la répartition au cours du traitement (Collier et al., 1976) ne semblent pas avoir d'influence significative sur les résultats. La quantité de réserpine administrée, compte tenu de ses effets secondaires potentiels, doit être limitée au maximum à deux fois $5 \mathrm{mg}$ par $24 \mathrm{~h}$. D'autres substances comme le sulpiride sont capables d'induire les mêmes effets sans provoquer les mêmes troubles (PonT J., résultats non publiés).

Bien que la sécrétion de prolactine présente des variations saisonnières importantes chez la Vache (Peters et al., 1981), les injections de réserpine pendant toute la durée des essais n'ont pas permis de supprimer les effets de la saison sur les productions laitières totales. Ces dernières sont plus élevées lorsque la durée d'éclairement quotidien augmente, c'est-à-dire quand la prolactinémie s'élève pendant la lactation normale (Peters et al., 1978) et la lactation après induction hormonale (Kessinger et al., 1979). Il est donc vraisemblable que même en présence de réserpine, les prolactinémies sont plus élevées lorsque les traitements d'induction sont réalisés au printemps. Il est possible que d'autres facteurs individuels (métabolisme) et de l'environnement (nutrition) influencent d'une façon saisonnière la production laitière après induction.

Le lait provenant de vaches induites en lactation a des taux normaux de matières grasses et de matières azotées.

Les hormones naturelles, utilisées pour induire la lactation (œstradiol $17 \beta$ et progestérone) s'éliminent très rapidement après l'arrêt du traitement (DeLouis et al., 1978). Les concentrations des cestrogènes totaux dans le lait produit par les vaches après vélage et après induction sont tout à fait comparables; celles de progestérone sont plus élevées pendant les 5 premiers jours qui suivent l'induction qu'après le vélage, cette différence disparaît en 10 jours (NARENDran et al., 1979). Il n'y a donc pas de risque de trouver des quantités anormales d'œstrogène et de progestérone dans les laits produits par les vaches après un traitement d'induction.

Aucune étude de l'influence des régimes nutritionnels sur la production laitière n'a été réalisée sur des vaches soumises au traitement d'induction de la lactation. Des échecs répétés, observés sur les animaux induits en lactation dans les mêmes exploitations peuvent, peut-être, s'expliquer par un régime alimentaire non adapté. La mise en 
place d'une lactation après induction ne bénéficie pas d'un état physiologique caractérisé par un haut niveau des réserves corporelles qui existe au cours de la montée laiteuse normale. Chez la Brebis, Leenanuruksa \& McDowell (1982) obtiennent une production laitière deux fois plus importante après perfusion continue de glucose pendant les 5 premiers jours d'une lactation induite. Chez la Vache, la production laitière normale est augmentée par une administration d'hormone de croissance (PEEL et al., 1983).

Les résultats des essais en ferme montrent qu'il est possible de remettre dans un cycle de reproduction des animaux réputés subfertiles avant le traitement d'induction ; toutefois, les résultats sont inférieurs à ceux obtenus au cours d'essais en station (tabl. 5b). Le manque de renseignements précis (état des ovaires, cyclicité) concernant les causes d'infertilité des animaux utilisés en ferme ne permet pas de proposer une explication pour ces différences de résultats ; pour des animaux présumés normaux, un délai d'entrée en gestation apparaît après traitement d'induction montrant ainsi clairement les effets défavorables de l'administration des hormones sur les performances de reproduction. Les fortes doses d'œstrogènes utilisées sont de nature à provoquer soit une stimulation, soit une inhibition de la fonction ovarienne selon l'état physiologique dans lequel se trouve l'animal (début ou fin de cycle, corps jaune persistant, follicule kystique, etc.) au moment du traitement d'induction. Les observations sur les performances de reproduction sont trop fragmentaires et portent sur un nombre trop faible d'animaux pour dégager une interprétation significative.

\section{Conclusion}

L'utilisation de la méthode d'induction de la lactation par un traitement hormonal de courte durée expérimentée dans les conditions de la pratique pourrait être appliquée comme méthode de production laitière pour des vaches non gestantes. Elle peut servir de sauvegarde avant la réforme d'animaux de très bon potentiel génétique, car les chances d'aboutir à un retour dans un cycle de reproduction des animaux traités sont de $30-40 \%$, à l'heure actuelle.

Elle pourrait également servir de méthode d'appréciation de la valeur laitière pour les animaux nullipares.

Actuellement, la méthode d'induction de la lactation dans les élevages en France est interdite par la législation très restrictive quant à l'apport d'œstrogènes à des animaux donnant des produits destinés à la consommation humaine. La présence d'œstrogènes et de progestérone en quantités normales dans le lait des vaches induites en lactation, liée à l'élimination rapide par l'animal des hormones naturelles injectées, autoriserait pourtant un développement de la méthode dans les conditions de la pratique.

Reçu pour publication en juin 1986.

Accepté en aô̂t 1987. 


\section{Remerciements}

Nous remercions les techniciens des Etablissements d'élevage des Départements et Régions que nous avons cités, ainsi que les vétérinaires et les éleveurs qui, par leur participation, ont rendu nos essais possibles. Nous remercions également $\mathbf{M}^{\mathrm{me}}$ A. Solari, de la Station de Physiologie animale, qui a effectué les analyses. statistiques des données.

\section{Summary}

\section{Hormonal induction of lactation in cows : results of three years of on-the-farm trials}

A hormonal short-term treatment was used to induce lactation in 363 dried non-pregnant dairy cows of different farms located in various regions in France.

Among the treated cows $76 \%$ produced at the minimum $10 \mathrm{~kg}$ milk d, but only $43 \%$ of the animals exhibited a lactation length over $200 \mathrm{~d}$ and the milk yield represented $69 \%$ of that of the previous normal lactation (tabl. 1). Total milk yields during induced lactations were significantly correlated with those recorded during previous $(r=0.38, P<0.02)$ and following $(r=0.39$, $\mathrm{P}<0.01$ ) control lactations in 46 cows (tabl. 4).

Protein contents of milk collected during induced lactations were significantly higher than those obtained during previous control lactations in the same 43 cows (3.34 vs $3.11 \%$ respectively, $P<0.01$ ). Fat contents of the same milk samples were not significantly different.

The lactation induction rates and milk yields obtained with hormonal treatments applied from January to July were significantly higher than those obtained from August to December ( 90 vs $71 \%$ and 4.242 vs $3.405 \mathrm{~kg} 305 \mathrm{~d}$, respectively) (tabl. 2 and 3 ).

Although lactation was induced in cows following reproductive failures, $38 \%$ of the animals became pregnant during induced lactation and calved (tabl. 5).

Induction of lactation could be used as a method for improving the profit earning capacity of high-yielding non-pregnant dairy cows during late lactation. However, because of the very strict legislation concerning the use of oestrogens it is not possible at the present time to further develop this technique in practice.

Key words : Induced lactation, reproduction, dairy cow, milk yield, field trials.

\section{Références bibliographiques}

Bauman D.E., Collier R.J., Tucker H.A., 1977. Effect of reserpine on serum prolactin, growth hormone and glucocorticoids in dairy cows. Proc. Soc. exp. Biol. Med., 155, 189-192.

Collier R.J., Bauman D.E., Hays R.L., 1975. Milk production and reproductive performance of cows hormonally induced into lactation. J. Dairy Sci., 58, 1524-1527.

Collier R.J., Bauman D.E., Hays R.L., 1976. Effect of reserpine on milk production and serum prolactin of cows hormonally induced into lactation. J. Dairy Sci, 60, 896-901.

Convey E.M., 1974. Serum hormone concentration in ruminants during mammary growth, lactogenesis and lactation. A review. J. Dairy Sci., 57, 905-914.

Davis S.R., Welch R.A.S., Pearce M.G., Peterson A.J., 1983. Induction of lactation in nonpregnant cows by estradiol $17 \beta$ and progesterone from an intravaginal sponge. $J$. Dairy Sci., 66, 450-457. 
Delouis C., 1980. Paramètres physiologiques de la formation et du fonctionnement de la mamelle. Journées de la Recherche ovine et caprine, INRA-I.T.O.V.I.C., pp. 5-34.

Delouis C., Ditane J., Kann G., Teroui M., Head H.H., 1978. Induced lactation in cows and heifers by short-term treatment with steroid hormones. Ann. Biol. anim. Bioch. Biophys., 18, 721-734.

Field D.E., McDowell G.P., Buesnel R.J., JesseP T.M., 1979. Artificial induction of lactation in unmated heifers and in heifers with reproductive abnormalities. Aust. J. Exp. Anim. Husb., $19,13-18$.

FULKERSON W.J., 1978. Artificial induction of lactation: A comparative study in heifers. Aust. J. Biol. Sci., 31, 65-71.

Garcia L., Rizo J.M., Martinet J., Delouis C., 1980. Induccion de la lactancia en vacas y novillas problemas. In "IX International Congress on animal Reproduction and A.I.", Vol. IV, pp. 381-386.

Johke T., Hodate K., Hodate K., Kawabata A., 1980. Effect of reserpine on plasma prolactin, growth hormone and thyrotropin in dairy heifers. Jap. J. Zootech. Sci., 51, 866-869.

Kensiger R.S., Bauman D.E., Collier R.J., 1979. Season and treatment effects on serum prolactin and milk yield during induced lactation. $J$. Dairy Sci., 62, 1880-1888.

LeEnanuruksa D., McDowell G.H., 1982. The importance of glucose supply at the initiation of artificial lactation in the ewe. Aust. J. Biol. Sci., 35, 629-636.

Mertes J., 1961. Farm animals. Hormonal induction of lactation and galactopoiesis. In " MILK : The mammary gland and its secretion " (S.K. Kon and A.T. CowIE, Eds), Vol. I, pp. 321-367, Acad. Press New-York.

De Montigny G., Pont J., Delouis C., 1980. L'induction de la lactation chez la chèvre. Bilan de 3 années d'utilisation dans les conditions de la pratique. Journées de la Recherche ovine et caprine, INRA-I.T.O.V.I.C., pp. 35-41.

Narendran R., Hacker R.R., Smith V.G., Lun A., 1979. Hormonal induction of lactation: estrogen and progesterone in milk. J. Dairy Sci., 62, 1069-1975.

Olds D., Cooper T., Thrift F.A., 1979. Relationships between milk yield and fertility in dairy cattle. J. Dairy Sci., 62, 1140-1144.

Peel C.J., Taylor J.W., Robinson I.B., Hooley R.D., 1979. The use oestrogen, progesterone and reserpine in the artificial induction of lactation in cattle. Aust. J. Biol. Sci., 32, 251-259.

Peel C.J., Fronk J., Bauman D.E., Gorewit R.D.C., 1983. Effect of exogenous growth hormone in early and late lactation on lactational performance of dairy cows. J. Dairy Sci, , 66, 776782 .

Peel C.J., Taylor J.W., Robinson I.B., McGowan A.A., Hooley R.D., Findlay J.K., 1978. The importance of prolactin in the milking stimulus in the artificial induction of lactation in cows. Aust. J. Biol. Sci., 31, 187-195.

Peters R.R., Chapin L.T., Emery R.S., Tucker H.A., 1981. Milk yield, feed intake, prolactin, growth hormone and glucocorticoid response of cows to supplemented light, J. Dairy Sci., 64, 1671-1678.

Peters R.R., Chapin L.T., Leining K.B., Tucker H.A., 1978. Supplemental lighting stimulates growth and lactation in cattle. Science, 199, 911-912.

Smith K., Schanbacher F.L., 1973. Hormone induced lactation in the bovine. Lactational performance following injections of $17 \beta$ oestradiol and progesterone. J. Dairy Sci., 56, 738743.

Smith K., Schanbacher F.L., 1974. Response of nulligravida heifers to modified estrogenprogesterone treatment. J. Dairy Sci., 57, 296-303. 